\title{
UN ANÁLISIS BIOGEOGRÁFICO DE LOS POLIQUETOS (ANNELIDA: POLYCHAETA) DEL GOLFO DE TEHUANTEPEC, MÉXICO
}

\author{
A BIOGEOGRAPHICAL ANALYSIS OF THE POLYCHAETES \\ (ANNELIDA: POLYCHAETA) FROM THE GULF OF TEHUANTEPEC, MEXICO
}

\author{
Vivianne Solís-Weiss' , Jesús Angel de León-González² \\ y Laura González-Ortíz'
}

\section{RESUMEN}

En esle trabajo se analizan las alinidades biogeográticas de 84 especies de anélidos poliquetos recolectados en la plataforma continental del golfo de Tehuantepec. Se encontraron cuatro componentes faunisticos dominantes: Amplia distribución (24,99\%), Anfiamericano (21,42\%). Pacífico $(42,84 \%)$ y Disyunto $(10,91 \%)$ definidos a partir de once grupos principales (Cosmopolita, Circuntropical, Anfiamericano Extendido, Anfiamericano en aguas cálidas, Pacífico Nororiental, Pacífico Oriental Tropical, Indopacífico, Transpacifico, Anfiamericano-Atlántico Oriental, Anfiamericano-Pacífico Occidental y Pacífico Oriental-Atlántico Oriental). Se discuten los patrones de distribución y la hipótesis de migración que pudieran explicar la distribucion de los anélidos poliquetos presentes en esa área, y se compara la distribución de los grupos principales con estudios de áreas cercanas del Pacífico, como Panamá, Colombia y la costa oeste de México.

Palabras Clave: Polychaeta, Biogeografia, Distribución, Golfo de Tehuantepec

\section{ABSTRACT}

The biogeographic affinities of 84 species of annelid polychaetes collected in the continental shelf of the Gulf of Tehuantepec are surveyed in this study. Four predominant faunistic assemblages were found: Wide distribution (24,99\%), Amphiamerican (21,42\%), Pacific $(42,84 \%)$ and Disjunct (10,91\%); those were defined from the eleven most generalized groups found there (Cosmopolitan, Circumtropical, Amphiamerican extended, Amphiamerican warm waters, Northeastern Pacific, Eastern tropical Pacific, Indopacific, Transpacific, Amphiamerican-Eastern Atlantic, Amphiamerican-Western Pacific, Eastern Pacific-Eastern Atlantic). The distribution patterns as well as the migration hypotheses that might explain the present distribution are discussed, and comparisons are made with recent biogeographic studies in nearby areas in the eastern Pacific such as Panama, Colombia and western Mexico.

Key words: Polychaeta, Biogeography, Distribution, Gulf of Tehuantepec.

\section{INTRODUCCIÓN}

Los anélidos poliquetos han sido consideradus frecuentemente un grupo cuyas especies no se ajustan a un patrón

' Laboratono de Ecologia Costera, Instituto de Cienclas del Mary Limnologia. Unwersiciad Nacional Autónoma de México. Aptoo. Postal 70-305, México. D. F. 04510. Mexico

2 Centro de Investigaciones Biológicas del Noroeste. S. C. y Laboratorio de Zoologia de Inventebrados. Fac. Ciencias Bióogicas. Universidad Autonoma de Nuevo León. Aptdo. Pustal 5 "F". San Nicalás de los Garza, N. L. 66451. México.

Correspondencia a

Dr. Jesus Angel de Ledn-Gonzalez.. Laboratoria de Zoologia de Invertebrados. Fac. Ciencias Biologicas, Universidad Autónoma de Nuevo Loón. Aptolo. Postal 5 "F". San Nicolás de los Garza, N. L 66451. México. biogeográfico convencional, como sucede con otros grupos de invertebrados marinos, por ejemplo los crustáceos, moluscos o equinodermos (San Martín 1984).

Por las dificultades que existen en el estudio de estos organismos (tamaño generalmente pequeño, posición inconspicua en el substrato, anatomía y taxonomía complicadas, no del todo resueltas), su etología y otras características que permiten definir afinidades biogeográficas son aún relativamente poco conocidas. Por 
ello, pocas han sido las aportaciones realizadas en el estudio de la biogeografía de poliquetos, entre las que destacan principalmente los trabajos de Cognetti (1955), Fauchald (1977), Holthe (1978), Bilyard y Carey (1979), San Martín (1984), Laverde-Castillo (1986), de León-González et al. (1993), Dauvin y Thiébaut (1994) y Kirkegaard (1994); asimismo, algunos autores han incluido en sus obras breves comentarios sobre el tema (Fauvel, 1923; Rioja, 1962a; Day, 1967; Imajima, 1967; Hartmann-Schröder, 1971).

Más recientemente, en una serie de trabajos realizados en México, se han propuesto algunas hipótesis tendientes a explicar las afinidades biogeográficas de las especies de poliquetos registradas en sus costas (Brusca, 1980; Salazar-Vallejo, 1985; Bastida-Zavala, 1991; Hernández-Alcántara, 1992; Salazar-Vallejo y Solís-Weiss, 1992; de León-González, 1994).

\section{MATERIAL Y MÉTODOS}

Las especies de poliquetos que se analizan en este trabajo fueron recolectadas durante la campaña oceanográfica MIMAR III (del Instituto de Ciencias del Mar y Limnología), realizada del 24 de mayo al 2 de junio de 1987 , a bordo del B/O "EL PUMA" (UNAM). Se muestrearon 50 localidades incluidas en 14 transectos a lo largo de la plataforma continental del Golfo de Tehuantepec, cuyas profundidades oscilaron entre 20 y $200 \mathrm{~m}$ (Fig. 1, Tabla 1). Las muestras se obtuvieron con una draga tipo Smith McIntyre de $0, \mathrm{I} \mathrm{m}^{2}$ y el sedimento fue tamizado a través de un tamiz de malla de $0,5 \mathrm{~mm}$.

Se obtuvieron los datos de distribución geográfica reportados previamente para las especies recolectadas en el Golfo de Tehuantepec, y se pudo determinar sus patrones de distribución, a partir de los cuales las especies se agruparon por similitud de sus respectivas distribuciones geográficas. Estas se compararon posteriormente con otros trabajos

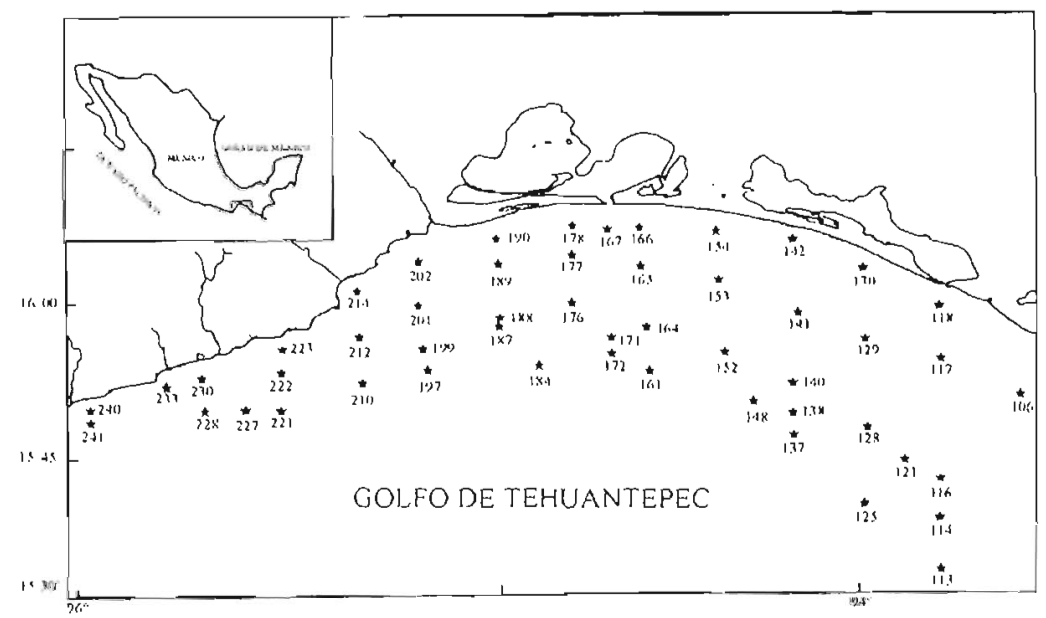

Figura 1. Mapa de distribución de las estaciones de colecta en el Golfo de Tehuantepec, México 
realizados en áreas cercanas al Golfo de Tehuantepec. La terminología utilizada para determinar los grupos fue tomada de Fauchald (1977) y Brusca (1980).

\section{RESULTADOS Y DISCUSIÓN}

Se estudiaron 1819 especímenes pertenecientes a 84 especies que fueron agrupadas en once categorías biogeográficas principales: Cosmopolita, Circuntropical, Anfiamericano Extendido, Anfiamericano en aguas cálidas, Pacífico Nororiental, Pacífico Oriental Tropical, Indopacífico, Transpacífico, Anfiamericano-Atlántico Oriental, Anfiamericano-Pacífico Occidental y Pacífico Oriental-Atlántico Oriental (Tabla 2). Se excluyó de este análisis a doce especies (229 organismos) recolectadas como parte del proyecto, que no se pudieron asignar a ninguna categoría, por no haberse podido determinar con certidumbre, ya sea por su mal estado o porque son nuevas para la ciencia y están en proceso de descripción.

Consideramos que estas once categorías pueden a su vez agruparse en cuatro componentes faunísticos dominantes: Amplia distribución, Anfiamericano, Pacífico y Disyunto, los que se definen y discuten a continuación.

En el componente "Amplia distribución", que constituye el $24,99 \%$ de las especies del estudio (Tabla 2), se incluyeron las formas cosmopolitas $(19,04 \%)$ que se distribuyen en aguas poco profundas de regiones templadas y frías por lo menos en los tres principales océanos, y las especies de distribución circuntropical $(5,95 \%)$, las cuales están presentes primordialmente en aguas cálidas.

El componente "anfiamericano", que constituye el $21,42 \%$ de estas especies (Tabla 2), como su nombre lo indica, está constituido por las especies que han sido regisradas en aguas de ambas costas del continente americano, sin importar la latitud $(15,47 \%)$ y las que se han reportado de aguas cálidas $(5,95 \%)$. Es interesante constatar que, a pesar de la latitud del área de estudio, encontramos que el porcentaje de este componente de especies previamente registradas en aguas calidas es menor que el de especies con más amplia distribución.

Desde el Oligoceno temprano hasta el Plioceno tardío, los océanos Atlántico y Pacífico estuvieron conectados a través del estrecho Panámico, lo cual favoreció el intercambio entre organismos de ambos cuerpos de agua (Valentine, 1971). Debido al transporte de larvas planctónicas por las corrientes ecuatoriales, las faunas de ambos océanos fueron relativamente similares hasta el Mioceno tardío (Woodring, 1966). En el caso de las formas del Golfo de Tehuantepec, era de esperarse que las especies anfiamericanas de aguas cálidas $(5,95 \%)$ prevalecieran sobre las de amplia distribución (o extendido) $(15,47 \%)$, pero los resultados encontrados (Tabla 2) difieren de esta suposición.

En el componente "Pacífico", que constituye el $42,84 \%$ del total, se incluyeron principalmente especies del Pacífico Nororiental $(17,85 \%)$ y del Pacífico Oriental Tropical $(17,85 \%)$, además de representantes de distribución transpacífica $(5,95 \%)$ e indopacífica (1,19\%), es decir, especies que han atravesado la barrera del Pacífico. Laverde-Castillo (1986) postula que esta travesía se pudo haber logrado por transporte en la contracorriente ecuatorial del norte, como ha sucedido con algunos moluscos, o bien, que han permanecido en el Pacífico Oriental desde que existía el mar de Tethys, y que sobrevivieron a las catástrofes del Pleistoceno en refugios, de manera similar a como lo pudieron haber hecho otros grupos animales.

Ahora bien, el concepto de que las especies pudieron ser transportadas a través de largas distancias por las corrientes oceánicas parece lúgico y aceptable; por ejemplo, una larva 
planctónica vive alrededor de 4 semanas, y si es arrastrada por una corriente de velocidad moderada a razón de $0,5 \mathrm{~km} / \mathrm{h}$, podría fijarse aproximadamente a una distancia de entre 300 y $350 \mathrm{~km}$ de su lugar de origen. Esto podría explicar los casos de especies con afinidades de tipo costero, pero resultaría imposible para explicar otro tipo de distribuciones, como la Transpacífica o Indopacífica (Salazar-Vallejo, 1985). Para estas últimas, se ha postulado clásicamente la colonización progresiva de las islas intermedias, hasta llegar a los márgenes costeros opuestos del Pacífico.

Otra posible explicación de su presencia en América, suponiendo 'nn origen en algún lugar del Pacífico Occidentu', está rclacionada con el posible acarreo por medios que las transportaran con parte de. o todo su hábitat. Se podría citar, por ejemplo, la utilización de frondas algales (Averincev, 1980), que permitirían un transporte por corrientes durante un ticmpo prolongado, dado que la(s) especie(s) invulucrada(s) puede(n) repetir todo su ciclo en numerosas ocasiones hasta la destrucción de estas frondas; también existen especics que se fijan a los cascos de embarcaciones, especialmente especies sésiles (Zibrowius, 1979) o, en el caso de los organismos no sésiles, que llegan a protegerse de la fricción directa del agua entre los intersticios u oquedades de la embarcación. Finalmentc, se han documentado casos en que la travesía se hace en el agua de los tanques de lastre de los mismos buques que se descarga directamente en las aguas del puerto de llegada, del otro lado del occano; en este caso, se trata generalmente cic las rases larvarias que pueden sobrevivir al viaje, para fijarse y colonizar el nuevo ambiente en que son depositadas (Carlton y Geller, 1993).

El componente "Disyunto", por su parte, que representa solamente el 10,91\% de las especies del estudio (Tabla 2), se compone de nueve especies cuyo patrón de distribución es irregular. En esta categoría se agruparon especies que han sido registradas tanto en ambas costas de América como en el Atlántico oriental (4,76\% de las especies del estudio), las especies cuya distribución previa conocida incluye el Pacífico oriental y el Atlántico oriental (que comprenden el 3,57\% del total), y por último las especies de distribución anfiamericana y Pacífico occidental, que en el Golfo de Tehuantepec están representadas por sólo el 2,58\% del total encontrado.

En su mayoría, las familias de poliquetos han sido registradas en todos los océanos y en todas las profundidades, y täinbién se ha observado en muchos de los géneros una amplia distribución. Esto ha originado que una proporción elevada de la fauna poliquetológica haya sido considerada generalmente cosmopolita (Fauvel, 1923, 1927, 1953; Day, 1967), extendiéndose esto a las especics del grupo, y agregando Hartman (1982) que el número de especies cosmopolilas es mayor cerca del Ecuador que en latitudes altas.

Sin embargo, cada vez hay más evidencias, y coincidimos con esto, de que a nivel específico, las especies declaradas cosmopolitas han sido mal identificadas y se trata en realidad de especies diferentes, aunque cercanas (Grassle y Grassle. 1976; SolísWeiss. el al., 1991)

Al comparar la composición biogeográfica de las especies de poliquetos del Golfo de Tuhuantepec con Ios resultados previos de los cstudios disponibles, realizados en el oeste de México, Panamá y Colombia (Tabla 3), se observa que las especies que se consideran cosmopolitas generalmente agrupan a la mayor parte de la fauna, excepto en el Golfo de California, cuyo caso se considera muy particular, por su morfología, en este estudio. Asimismo, los trabajos de Fauchald (1977) para Panamá y de Laverde-Castillo (1986) para el Pacífico colormbiano difieren de 10 expuesto por Hartman (1982).

La presencia de especies con distribución Pacífico Nororiental disminuye notablemente 
hacia el Ecuador y se observa que en el Golfo de California (Kudenov, 1980), el 36\% de las especies se agrupan en esta categoría, y en cambio en el Pacífico colombiano (Laverde Castillo, 1986) sólo el $1 \%$ pertenece a este grupo. En nuestro caso, se encontró el 17,85\% de las especies bajo ciste patrón biogeográfico.

La fauna correspondiente al componente "anfiamericano" se encuentra representada por el $21,42 \%$ de la colecta, resultado que es muy similar a los de estudios anteriores en el Pacífico colombiano (32\%) (Laverde Castillo, 1986) y el Golfo de California (25\%) (Hernández-Alcántara, 1992). Sin embargo, es superior a los porcentajes encontrados en la plataforma continental de la costa oeste de Baja California Sur (12\%) (de León-González, 1994), las bahías de la Paz (10\%) (BastidaZavala, 1992) y Concepción (11\%) (SalazarVallejo, 1985). Lo mismo sucede en el estudio de Kudenov (1980) (3\%), que representa básicamente a la fauna intermareal, asociada predominantemente a la región del Pacífico Nororiental. Fislo podría explicarse mediante el concepto del flujo biótico transístmico (Rosen, 1975). La aceptación de este concepto implica que, antes del cierre del paso de Panamá, existía un gran grupo de plantas y animales que se distribuían en ambas costas tropicales: del Pacífico Oriental y del Atlántico Occidental. Algunas otras estimaciones sugieren que entre el $5 \%$ y $40 \%$ de las especies de invertebrados del Pacífico Este Tropical podrían tener especies análogas en el Atlántico (dependiendo del grupo), lo que acentúa la afinidad biológica de estas regiones.

Los grupos Pacífico Nororiental, Pacífico Oriental Tropical y Anfiamericano agrupan al $56 \%$ del total de las especies de poliquetos determinadas en el golfo de Tehuantepec, de tal modo que se puede considerar que tenemos aquí representada a una fauna predominantemente "americana", ya que las especies de dicho grupo sólo se han registrado hasta el momento en este continente. Con esto se refuerza la hipótesis de Day (1967), quien postula que la fauna poliquetológica del "Nuevo Mundo" podría ser distinta a la europea y muy posiblemente diferente de la de otros continentes.

\section{AGRADECIMIENTOS}

Agradecemos al Dr. Arturo Carranza E. responsable del proyecto MIMAR, a la tripulación del B/O El Puma, y al personal científico participante en la campaña MIMAR III por la ayuda prestada en la recolección de las muestras, así como a los compañeros del laboratorio de Ecología Costera que colaboraron en este proyecto, en especial al Técnico Académico Pablo Hernández Alcántara, por sus acertados comentarios.

\section{LITERATURA CITADA}

Averincev, V. G. 1980. The role of drifting islands of seaweeds in the distribution of bottom polychaetes. Biol. Morya 1: 80-83.

Bastida-Zavala, J. R. 1991. Poliquetos (Annelida: Polychaeta) del sureste de la Bahía de La Paz, B. C. S., México: Taxonomía y aspectos biogeográficos Tesis Prof. UABCS, 158 pp.

Bilyard, G. R. y A. G. Carey. 1979. Distribution of Western Beaufort Sea Polychaetous Annelids. Mar. Biol. 54: 329-339.

Brusca, R. C. 1980. Common intertidal invertebrates of the Gulf of California. 2nd ed., Univ. Arizona Press, Tucson, $513 \mathrm{pp}$.

Carlton, J. T. y J. B. Geller. 1993. Ecological Raulette: The Global Transport of nonindigenous Marine Organisms. Science 261: 78-82. 
Cognetti, G. 1955. I sillidi dal punto de vista ecologico e zoogeografico. Bull, Zool. 22: $225-228$.

Dauvin, J. C. y E. Thiébaut. 1994. Is Owenia fusiformis a cosmopolitan species? Mem. Mus. Nat. Hist. Natur. Paris, 162 : 383-404.

Day, J. H. 1967. A monograph on the Polychaeta of Southern Africa. Brit. Mus. (Nat. Hist.) Publ. 656: 1-878

de León-González, J. A. 1994. Poliquetos (Annelida: Polychaeta) de la plataforma continental de la costa oeste de Baja California Sur, México: Taxonomía, hábitos alimenticios y distribución. Tes. Maestr., CICIMAR-IPN, $177 \mathrm{pp}$.

de León-González, J . A., A. Leija-Tristán y S I. Salazar-Vallejo. 1993. Epifauna del ostión espinoso Spondylus princeps unicolor (Mollusca: Bivalvia), de Puerto Escondido, Golfo de Califormia, México. Rev. Biol. Trop. 4l(3): 877-881.

Fauchald, K. 1977. Polychaetes from intertidal areas in Panama, with a review of previous shallow-water records. Smithson. Contr. Zool. 221: 1-85

Fauve1, P. 1923. Polychètes Errantes. Faune de France 5: 1-488

Fauvel, P. 1927. Polychètes Sédentaires et Addenda aux Polychètes Errantes, Archiannélides, Myzostomaires. Ibíd. 16: $1-494$

Fauvel, P. 1953. The Fauna of India including Pakistan, Ceylon, Burma and Malaya. Annelida Polychaeta. Indian Press, Allahabad, 519 pp.
González-Ortíz, L. 1994. Los poliquetos (Annelida: Polychaeta) de la plataforma continental del Golfo de Tehuantepec, México. Tesis Prof., Fac. Ciencias, UNAM, $191 \mathrm{pp}$.

González-Ortíz, L., P. Hernández-Alcántara y V. Solís-Weiss. 1996. New records of polychaetes (Annelida: Polychaeta) from the Gulf of Tehuantepec. Bull. Mar. Sci. 59(1): 235-240.

Grassle, J. y F. Grassle. 1976. Sibling species in the marine pollution indicator Capitella (Polychaeta). Science 192: 567-569

Hartman, O. 1982. Polychaeta, pp. 615-619. On Mc Graw-Hill Encyclopedia of Science and Technology. 5th edition. Mc Graw-Hill Company, New York

Hartmann-Schröder, G. 1971. Annelida, Borstenwürmer, Polychaeta. Tierwelt Deutschlands 58: 1-594

Hernández-Alcántara, P. 1992. Los poliquetos (Annelida: Polychaeta) de la plataforma continental del Golfo de California, México. Taxonomía, abundancia numérica y distribución geográfica. Tesis de Maestría. ICM y L, UACP y $\mathrm{P}-\mathrm{CCH}$, UNAM, 427 pp.

Holthe, T. 1978. The zoogeography of the Terebellomorpha (Polychaeta) of the Northern European waters. Sarsia 63: 191-198

Imajima, M. 1967. Errant polychaetous annelids from Tsukamo Bay and vicinity of Noto peninsula, Japan. Bull. Natl. Sci. Mus. 10: 403-441.

Kirkegaard, J.B. 1994. The biogeography of some abyssal polychaetes. Mem. Mus. Nat. I-Iist. Natur. Paris, 162: 471-478. 
Kudenov, J. D. 1980. Annelida: Polychaeta (Bristleworms). pp: 77-123. En R.C. Brusca (ed.): Common Intertidal Invertebrates of the Gulf of California, 2nd ed., Univ. Arizona Press, Tucson, $715 \mathrm{pp}$.

Laverde-Castillo, J. J. A. 1986. Lista anotada de los poliquetos (Annelida) registrados para el Pacífico Colombiano, con notas preliminares sobre su zoogeografía. Act. Biol. 15(58): 123-130.

Rioja, E. 1962a. Estudios Anelidológicos XXVI. Algunos anélidos poliquetos de las costas del Pacífico de México. Ibíd. 33: 131-229

Rosen, D. E. 1975. A vicariance model of Caribbean biogeography. Syst. Zool. 24(4): 43 l -464

Salazar-Vallejo, S. I. 1985. Contribución al conocimiento de los poliquetos (Annelida: Polychaeta) de Bahía Concepción, Baja California Sur, México. Tesis Maestría, CICESE, $31.1 \mathrm{pp}$.

Salazar-Vallejo, S. I. y V. Solís-Weiss. 1992. Biogeography of the pilargid polychaetes (Polychaeta: Pilargidae) of the Subfamily Synelminae, pp. 273-284.
En S. P. Darwin y A. L. Welden (eds.). Biogeography of Mesoamerica. Proc. Symposium, Mérida, México, oct. 1984. Tulane Stud. Zool. Bot., Suppl. Publ. 1 (mayo 1992).

San Martín, G. 1984. Biogeography of the Syllidae (Polychaeta) from the Spanish Mediterranean Coasts. Proc. First Internat. Polychaete Conf., Sydney, ed by P.A. Hutchings, The linnean Soc. New South Wales, pp. 303-322.

Solís-Weiss, V., K. Fauchald y A Blankesteiyn. 1991. Trichobranchidae (Polychaeta) from shallow warm water areas in the Western Atlantic Ocean Proc. Biol. Soc. Wash. 104(1): 147-158.

Valentine, J. W. 1971. Plate tectonics, shallow marine diversity and endemism, an actualistic model. Syst. Zool, 20: 253-264.

Woodring, W. P. 1966. The Panama land bridge as a sea barrier. Proc. Am. Phil. Soc. 110:425-433.

Zibrowius, H. 1979. Serpulidae (Annelida, Polychaeta) de l'Océan Indien arrivés sur des coques de bateaux à Toulon (France, Méditerranée). Rapp. Comm. int. mer medit. 25/26 (4): 132-133. 
Tabla 1. Lista anotada de los poliquetos del Golfo de Tehuantepec con el patrón de distribución: Cosmopolita (COS), Circuntropical (CTR), Pacífico Nororiental (PNO), Pacífico Este Tropical (PET), Anfiamericano extendido (ANX), Anfiamericano en aguas cálidas (ANC), Indopacífico (IND), Transpacifico (TRA), Disyunto Anfiamericano-Atlántico Oriental (DAA), Disyunto Anfiamericano-Pacífico Occidental (DAP), Disyunto Pacifico Oriental-Atlántico-Oriental (DPA).

\section{Especie}

Orbiniidae

Leitoscoloplos mexicanus (Fauchald)

L. panamensis (Morro)

L. pugettensis (Pettibone)

Scoloplos (Leodamas) ohlini (Ehlers)

Scoloplos cf. capensis

Paraonidae

Aricidea (Acmira) lopezi Berkeley y Berkeley

Aricidno (Aedecira) pacifica Hartman

Aricidea (Allia) suecica Eliason

Aricidea (Aricidea) fragilis Webster

Aricidea (Aricidea) longicirrata Hartmann-Schroder

Cirrophorus furcatus (Hartman)

Paradoneis lyra (Southern)

Levinsenia gracilis (Tauber)

Cossuridae

Cossura brunnea Fauchald

Spionidae

Apoprionospio pygmaea (Hartman)

Paraprionospio pinnata (Ehlers)

Prionospio (Minuspio) delta Hartman

Prionospio (Minuspio) lighti Maciolek

Prionospio (Prionospio) steenstrupi Malmgren

Rhynchospio glutaeus (Ehlers)

Scolelepis (Parascolelepis) texana (Foster)

Scolelepis (Scolelepis) squamata (Muller)

Spio uncinata Hartman

Spiophanes wigleyi Pettibone

Magelonidae

Magelona californica Hartman

Magelona pacifica Monro

Magelona pitelkai Hartman

Poecilochactidae

Poecilochaetus johnsoni Hartman
COS

TRA

COS

PET

Patrón

PET

PET

TRA

TRA

DAA

CTR

PET

PNE

ANX

COS

ANX

COS

DAA

PNE

COS

TRA

ANX

PNE

ANC

DAP

PNE

TRA

PNE

PNE

Continúa... 
Viene Tabla 1

Cirratulidae

Aphelochaeta multifilis Moore

IND

Aphelochaeta parvus Berkeley

PNE

Aphelochaeta serraticetis (Banse y Hobson)

PNE

Chaetozone setosa Malmgren

COS

Monticellina tesselata (Hartman)

PET

Capitellidae

Notomastus daueri (Ewing)

ANC

Notomastus hemipodus (Hartman)

ANX

Opheliidae

Armandia agilis (Anderws)

ANX

Armandia intermedia Fauve]

CTR

Ophelia assimilis Tebble

PNE

Ophelia pulchella Tebble

PNE

Ophelina acumunata Orsted

COS

Eulepethidae

Grubeulepis mexicana (Berkeley y Berkeley)

ANC

Sigalionidae

Sigalion lewissi (Berkeley y Berkeley)

ANC

Sthenolepis fimbriarum. (Hartman)

PET

Pilargidae

Loandalia salazarvallejoi de León-González

PET

Sigambra tentaculata (Treadwell)

COS

Syllidae

Ehlersia cornuta (Rathke)

COS

Typosyllis prolifera (Krohn)

CTR

Nereididae

Ceratocephale oculata Banse

ANX

Gymnonereis crosslandi (Monro)

ANC

Neanthes succinea (Frey y Leuckart)

COS

Nereis angelensis Fauchald

PET

Nereis pelagica Linnaeus

$\operatorname{COS}$

Glyceridae

Glycera convoluta Keferstein DPA

Glycera papillosa Grube DAA

Glycera resselatu Grube CTR

Goniadidae

Glycinde solitaria (Webster)

ANX

Goniada maculata Orsted 
Solís-Weiss, De León-González y González-Ortíz

Viene tabla 1

Nephtyidae

Aglaophamus verrilli (McIntosh)

$\mathrm{ANC}$

Nephtys magellanica Augener

$\mathrm{ANX}$

Nephtys parva Clark y Jones

PNE

Amphinomidae

Chloeia viridis (Schmarda)

PET

Linopherus ambigua (Monro)

ANX

Onuphidae

Diopatra obliqua Hartman

PET

Diopatra ornata Moore

PNE

Diopatra splendidissima Kinberg

PET

Diopatra tridentata Hartman

ANX

Mooreonuphis peruana (Hartman)

PET

Eunicidae

Eunice vittata (Delle Chiaje)

$\operatorname{COS}$

Lumbrineridae

Lumbrineris crassidentata Fauchald

PET

Lumbrineris latreilli Audouin y Milne-Edwards

COS

Lumbrineris limicola Hartman

PNE

Lumbrineris platylobata Fauchald

PET

Lumbrineris tetraura (Schmarda)

ANX

Lumbrineris zonata (Johnson)

PNE

Ninoe foliosa Fauchald

PET

Oenonidae

Arabella iricolor (Montagu)

$\operatorname{COS}$

Flabelligeridae

Brada villosa (Rathke)

$\operatorname{COS}$

Ampharetidae

Amphicteis scaphobranchiata Moore

ANX

Eclysippe vanelli (Fauvel)

DPA

Isolda pulchella Müller

CTR

Terebellidae

Pista quadrilobata (Augener)

DAA

Sabellidae

Jasmineira pacifica Annenkova

DAP

Potamilla neglecta (Sars)

DAP

Potamethus mucronatus (Moore)

PNE 
Tabla 2. Patrones de distribución de 84 especies de poliquetos registrados para el Golfo de Tehuantepec.

\section{Patrón}

1. Ampliamente distribuidos

- Cosmopolita

- Circuntropical

2. Anfiamericano

- Extendido

- Aguas cálidas

3. Pacífico

- Pacífico nororiental

- Pacífico oriental tropical

- Indopacífico

- Transpacífico

4. Disyunto

- Anfiamericano-atlántico oriental

- Anfiamericano-pacífico occidental

- Pacífico oriental-atlántico oriental

\section{Especies}

N. ${ }^{\circ}$ $\%$

$16 \quad 19,04$

$5 \quad 5,95$

$13 \quad 15,47$

$5 \quad 5,95$

$15 \quad 17,85$

$15 \quad 17,85$

$1 \quad 1,19$

$5 \quad 5,95$

Tabla 3. Composición biogeográfica porcentual de diferentes taxocenosis regionales: Fauchald 1977 (Panamá), Laverde-Castillo 1986 (Pacífico Colombiano), Kudenov 1980 (Golfo de California), Salazar-Vallejo 1985 (Bahía Concepción, México), Bastida-Zavala 1991 (Bahia de La Paz, México), Hernández-Alcántara 1992 (Golfo de California), de León-González 1994 (Costa oeste de Baja California Sur, México). Cosmopolita (COS), Circuntropical (CTR), Pacífico Nororiental (PNO), Pacífico Oriental Tropical (POT), Anfiamericano (ANF), Indopacífico (IND), Transpacífico (TRA), Endémico (END).

\section{COS CTR PNO POT ANF IND TRA END}

$\begin{array}{lcccccccc}\text { Fauchald I977 } & 10 & 21 & 9 & 21 & 16 & 5 & - & 12 \\ \text { Laverde Castillo 1986 } & 12 & 20 & 1 & 27 & 32 & 2 & - & - \\ \text { Kudenov 1980 } & 17 & - & 36 & 9 & 3 & - & - & 28 \\ \text { Salazar-Vallejo 1985 } & 31 & 14 & 14 & 13 & 11 & - & 10 & 8 \\ \text { Bastida-Zavala 1991 } & 21 & 12 & 22 & 7 & 10 & 6 & - & 16 \\ \text { Hernández-Alcántara 1992 } & 26 & - & 20 & 7 & 25 & - & 5 & 9 \\ \text { de León-González 1994 } & 20 & 1 & 9 & 32 & 12 & 5 & 9 & 7\end{array}$

